\title{
Clinical Efficacy and Safety of Thai Herbal Formulation-6 in the Treatment of Symptomatic Osteoarthritis of the Knee: A Randomized-Controlled Trial
}

\author{
Nut Koonrungsesomboon $\left(\mathbb{D D}^{1,2}\right.$ Saowaros Nopnithipat, ${ }^{1}$ Supanimit Teekachunhatean, ${ }^{1,3}$ \\ Natthakarn Chiranthanut, ${ }^{1,3}$ Chaichan Sangdee, ${ }^{1}$ Sunee Chansakaow, ${ }^{4}$ \\ Pramote Tipduangta, ${ }^{4}$ and Nutthiya Hanprasertpong ${ }^{1}{ }^{1}$ \\ ${ }^{1}$ Department of Pharmacology, Faculty of Medicine, Chiang Mai University, Chiang Mai 50200, Thailand \\ ${ }^{2}$ Musculoskeletal Science and Translational Research Center, Chiang Mai University, Chiang Mai 50200, Thailand \\ ${ }^{3}$ Center of Thai Traditional and Complementary Medicine, Faculty of Medicine, Chiang Mai University, \\ Chiang Mai 50200, Thailand \\ ${ }^{4}$ Department of Pharmaceutical Sciences and Medicinal Plant Innovation Center, Faculty of Pharmacy, Chiang Mai University, \\ Chiang Mai 50200, Thailand
}

Correspondence should be addressed to Nutthiya Hanprasertpong; nutthiya.h@cmu.ac.th

Received 3 September 2020; Revised 24 October 2020; Accepted 3 November 2020; Published 9 December 2020

Academic Editor: Arham Shabbir

Copyright (C) 2020 Nut Koonrungsesomboon et al. This is an open access article distributed under the Creative Commons Attribution License, which permits unrestricted use, distribution, and reproduction in any medium, provided the original work is properly cited.

\begin{abstract}
Background. Osteoarthritis of the knee is the most common form of arthritis. Identifying effective and safe herbal formulations that are locally available is viewed as a priority for sustainable development in a region. This study aimed to evaluate the efficacy and safety of Thai herbal formulation-6 (THF-6) in comparison with oral diclofenac in patients with moderate-to-severe osteoarthritis of the knee. Methods. This randomized, double-blind, active-controlled, noninferiority trial randomly assigned patients with osteoarthritis of the knee to receive either THF- 6 or diclofenac for four weeks. The primary outcome measure was the change from baseline in knee pain as measured by a $100 \mathrm{~mm}$ visual analog scale (VAS). Secondary outcome measures included knee stiffness, a stair climb test, the Knee Injury and Osteoarthritis Outcome Score, and safety parameters. Outcomes were assessed on a biweekly basis. Modified intention-to-treat (MITT) and perprotocol (PP) analyses were applied. Results. A total of 200 patients were enrolled of whom 175 (87.5\%) were included in the MITT analysis and 153 (76.5\%) in the PP analysis. The mean change in VAS pain did not differ between the two groups, and the upper limit of the two-sided $95 \%$ confidence interval (CI) for comparison between the two groups was within the prespecified margin of $10 \mathrm{~mm}$ for noninferiority (MITT analysis: mean difference $=0.86,95 \% \mathrm{CI}=-4.39$ to $6.10, p=0.748$; $\mathrm{PP}$ analysis: mean difference $=1.98,95 \% \mathrm{CI}=-3.61$ to $7.56, p=0.486$ ). Significant improvement was observed in all the efficacy parameters in both groups. Dyspepsia was the most common adverse event: 23 patients in the THF-6 group and 28 in the diclofenac group $(p=0.417)$. Conclusions. THF- 6 offers an alternative to oral diclofenac for the short-term treatment of osteoarthritis of the knee. It was shown to be noninferior to oral diclofenac in relieving knee pain. This trial is registered with ChiCTR-IPR-15007213.
\end{abstract}

\section{Introduction}

Osteoarthritis is the most common form of arthritis, commonly affecting knee joints and causing pain, functional disability, and reduced quality of life $[1,2]$. Osteoarthritis of the knee is one of the leading causes of global disability among older adults [3]. The burden of disease on the healthcare system, in addition to its impacts on individual patients, has been increasing worldwide over the past three decades $[4,5]$. Nearly half of the population may develop symptomatic osteoarthritis of the knee by age 85 , and the lifetime risk is doubled among obese individuals [6]. With 
increasing life expectancy, osteoarthritis of the knee is anticipated to cause even more economic challenges in the future that every country and international community needs to grapple with [7].

Nonsteroidal anti-inflammatory drugs (NSAIDs) are one of the most widely used medications for the management of knee pain in patients with osteoarthritis of the knee [8]. The efficacy of oral NSAIDs, diclofenac in particular, for symptomatic relief of knee osteoarthritis has been well established in the literature [9]. Thanks to their good analgesic and antiphlogistic effects, NSAIDs are recommended by several international and national guidelines as the initial oral medication of choice in the treatment of symptomatic osteoarthritis of the knee with moderate-to-severe pain intensity $[10,11]$. Several NSAIDs, including oral diclofenac, are available as over-the-counter medications in many countries, so the use of this drug is particularly widespread $[12,13]$.

Given the large number of people affected with osteoarthritis of the knee and societal trends in population aging and obesity [14], identifying effective and safe therapeutic options that are available in a region is viewed as a priority for sustainable development in a country [15]. In this regard, herbal medicine could be a promising approach to be employed in developing such therapeutic options [16]. Several herbal extracts and formulations have been discovered and proven that they can bring about therapeutic benefits in terms of pain and mobility in patients with osteoarthritis of the knee [17-19]. In Thailand, the Thai herbal formulation-6 (THF-6) comprising six herbal materials, all of which are locally available in Thailand, has traditionally been used to augment longevity as well as for the treatment of muscle and joint pain [20-22]. Although anecdotal evidence exists supporting the efficacy and tolerability of THF- 6 in the treatment of osteoarthritis of the knee [22], scientific proof of its benefits is required to verify its therapeutic potential.

The present study was designed to clinically assess the efficacy and safety of THF- 6 in comparison with the standard drug, oral diclofenac, for the treatment of moderate-tosevere osteoarthritis of the knee.

\section{Methods}

2.1. Trial Design and Setting. This prospective, randomized, double-blind, double-dummy, and active-controlled trial was conducted at the Faculty of Medicine, Chiang Mai University, Thailand. The trial followed the OARSI Clinical Trials recommendations for the design, conduct, and reporting of clinical trials for osteoarthritis of the knee [23]. It was prospectively registered with the Chinese Clinical Trials Registry (ChiCTR-IPR-15007213). The clinical trial protocol and related documents were approved by the Research Ethics Committee of the Faculty of Medicine, Chiang Mai University (EC273/2015). The fulltrial protocol (in Thai) is available from the corresponding author upon reasonable request. Written informed consent was obtained from all patients prior to their participation in the trial.
2.2. Trial Participants. Eligible patients were 45 years of age or older with osteoarthritis in one or both knees for more than three months. The disease was diagnosed according to the American College of Rheumatology criteria [24] with radiographic confirmation (Kellgren-Lawrence grade 2 or higher). Patients were eligible for inclusion if they had osteoarthritic knee pain of at least moderate intensity (defined as a pain score of $\geq 35 \mathrm{~mm}$ at baseline on a $100 \mathrm{~mm}$ visual analog scale (VAS)) [25]. Patients were excluded if they also had any other underlying arthritis (e.g., rheumatoid arthritis or gouty arthritis), signs or symptoms of active inflammation at the knee, a condition requiring knee surgery in the next few months, or a recent knee injury; had used intraarticular corticosteroid injections in the previous six weeks; had used symptomatic slow-acting drugs for osteoarthritis (e.g., glucosamine sulfate or chondroitin sulfate) within the previous four months or had discontinued those drugs for less than six months; had a history of an allergic reaction to oral NSAIDs or herbal ingredients in THF-6; or had a history of gastrointestinal ulcer, perforation, or hemorrhage. Other exclusion criteria were pregnancy or lactation and any clinically significant abnormalities of blood chemistry (including serum uric acid of $>9 \mathrm{mg} / \mathrm{dL}$ ) or other hematological parameters.

2.3. Sample Size Determination. With 69 patients per group, the trial was estimated to have $90 \%$ power at a two-sided alpha level of 0.05 based on a noninferiority margin of 10 $[26,27]$ and assuming a mean difference (MD) of 0 and a standard deviation (SD) of 20 [28]. Anticipating a 30\% premature discontinuation or poor compliance, a total of 200 patients (100 per group) were planned to be enrolled in this trial.

2.4. Trial Interventions. The THF-6 (given at the dose of $1,500 \mathrm{mg} /$ day) consisted of six herbs: fruit of Streblus asper Lour, stems of Tinospora cordifolia (Willd.) Miers, corms of Cyperus rotundus, bark of Albizia procera (Roxb.) Benth, bark of Diospyros rhodocalyx Kurz, and fruit of Piper nigrum Linn [21]. The components and preparation of THF-6 are summarized in Table S1. The THF-6 capsules $(250 \mathrm{mg} /$ capsule) were manufactured by the Department of Pharmaceutical Sciences and Medicinal Plant Innovation Center, Faculty of Pharmacy, Chiang Mai University, in compliance with the Thai Pharmacopoeia standards and requirements. High-performance liquid chromatography and thin-layer chromatography were used for quality control of THF-6.

Diclofenac (Voltaren $\left.{ }^{\circledR}\right)(25 \mathrm{mg} /$ tablet$)$ was purchased from Olic Thailand limited and used as an active control in this trial. Placebo tablets of THF-6 and diclofenac were manufactured by the Department of Pharmaceutical Sciences and Medicinal Plant Innovation Center, Faculty of Pharmacy, Chiang Mai University.

2.5. Trial Procedures and Outcome Assessments. The trial consisted of a one-week run-in phase followed by a fourweek treatment phase (Figure 1). During the one-week run- 


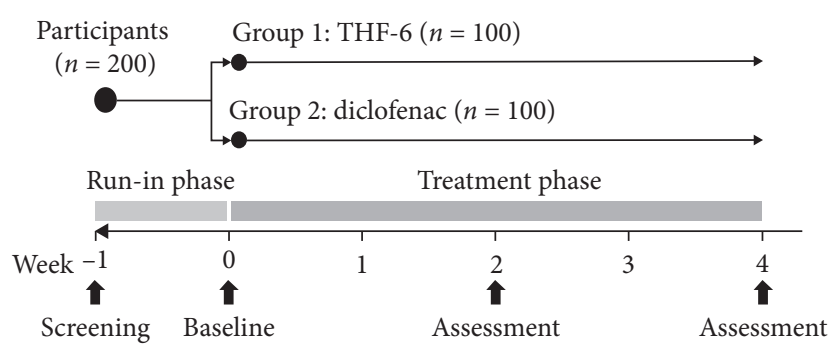

Figure 1: Study design.

in period, eligible patients were instructed to discontinue all pain relief medications (including NSAIDs and other analgesics). At the beginning of the treatment phase, eligible patients with at least moderate pain intensity were randomly assigned in a $1: 1$ ratio to receive either THF- $6(1,500 \mathrm{mg} /$ day) or diclofenac $(75 \mathrm{mg} /$ day) (Figure 1). The treatment group assignment was based on a randomization list prepared in advance by independent research staff with the use of a computer-based random number generator. Sequentially numbered, opaque envelopes containing the list were employed to safeguard allocation concealment. The envelopes were opened only after each patient had met the eligibility criteria at the end of the run-in phase. Both patients and outcome assessors were blinded to the treatment allocation.

In the THF- 6 group, patients took two capsules of THF- 6 $(250 \mathrm{mg} / \mathrm{capsule})$ and one placebo diclofenac tablet three times a day after meals. In the diclofenac group, patients took two placebo THF- 6 capsules and one diclofenac tablet $(25 \mathrm{mg} /$ tablet$)$ three times a day after meals. Enrolled patients were instructed to avoid other analgesics, anti-inflammatory drugs (including other NSAIDs), and other treatment modalities (e.g., acupuncture) while participating in the trial. Omeprazole tablets $(20 \mathrm{mg} / \mathrm{tablet})$ were prepared as rescue therapy in case of adverse gastrointestinal consequences following the administration of the study drugs. Patients were prematurely withdrawn from the trial if they developed intolerable knee pain necessitating other medications or treatment modalities, used other analgesics or anti-inflammatory drugs, had severe adverse drug reactions or allergic reactions to the study drugs, or were lost to follow-up.

This trial assessed efficacy outcomes for pain, function, and global assessment according to the recommendations of a core domain set for outcome measurement in clinical trials of knee osteoarthritis $[29,30]$. Outcome assessment was performed on a biweekly basis, that is, at the end of the oneweek run-in phase (baseline) and at the end of Week 2 and Week 4 (Figure 1).

The primary efficacy metric was the change from baseline in knee pain as measured by a horizontal $100 \mathrm{~mm}$ VAS (rated on a scale of 0 to 100, with higher scores indicating worse knee pain) [31]. The secondary efficacy outcomes included VAS stiffness (rated on a scale of 0 to 100 , with higher scores indicating more severe stiffness), a 10step stair climb test (SCT) (time in seconds to ascend and descend a flight of 10 steps) [32], the Knee Injury and
Osteoarthritis Outcome Score (KOOS) (which included 42 items across five domains; each item was rated on a 5-point Likert scale and transformed to a scale of 0 to 100 , with higher scores indicating fewer knee problems) [33, 34], and the patient's and the physician's opinions of overall improvement on a $100 \mathrm{~mm}$ VAS (rated on a scale of 0 to 100 , with higher scores indicating better improvement). In patients with bilateral osteoarthritis of the knee, efficacy outcomes were assessed only for the knee with worse symptoms at baseline.

Adverse events observed by the investigators or reported by the patients following a nondirective question were recorded. Drug compliance was assessed by counting returned unused medications at each visit. Any patient taking less than $70 \%$ of the allocated dose of study drugs was regarded as noncompliant.

2.6. Statistical Analysis. Analyses of efficacy outcomes were conducted using the perprotocol (PP) and the modified intention-to-treat (MITT) approaches, with the last observation carried forward method. For the safety evaluation, all patients who had received at least one dose of study drugs were analyzed. Statistical analysis was performed using SPSS version 22.0. A $p$ value of $<0.05$ was considered to indicate statistical significance.

Continuous variables are presented as mean \pm SD. Within-group comparisons were conducted to determine any differences in the mean values of each variable between baseline and the two consecutive follow-up visits; a repeated measures ANOVA, with the least significant difference (LSD) test, was applied. For between-group comparisons, mean changes from baseline were compared using Student's $t$-test. Patients were classified as having had a response if their VAS pain scores decreased by $50 \%$ or more from baseline [35]. Dichotomous variables are reported as frequencies; the chi-squared test or Fisher's exact test, as appropriate, was used to compare the distribution of dichotomous variables between the two groups.

For assessment of noninferiority, a comparison between the THF- 6 group and the diclofenac group on VAS pain was conducted, with a prespecified noninferiority margin of $10 \mathrm{~mm}[26,27]$. Noninferiority was declared if the upper limit of the two-sided 95\% confidence interval (CI) for the MD of VAS pain did not exceed a margin of $10 \mathrm{~mm}$.

\section{Results}

From February 2016 to July 2016, a total of 349 patients were screened for eligibility and 200 underwent randomization, 100 of whom were allocated to each arm (Figure 2). Predominant reasons for screening failure were that the patient did not meet the inclusion criteria (115 patients, $77.2 \%$ ) and that the patient had abnormal laboratory results (31 patients, $20.8 \%)$. The mean age of the enrolled patients was $61.1 \pm 6.1$ years; $85.5 \%$ were women and $86 \%$ had osteoarthritis in both knees. Baseline demographic and clinical characteristics of the patients were comparable between the two groups (Table 1). Of the 200 patients randomized, 175 


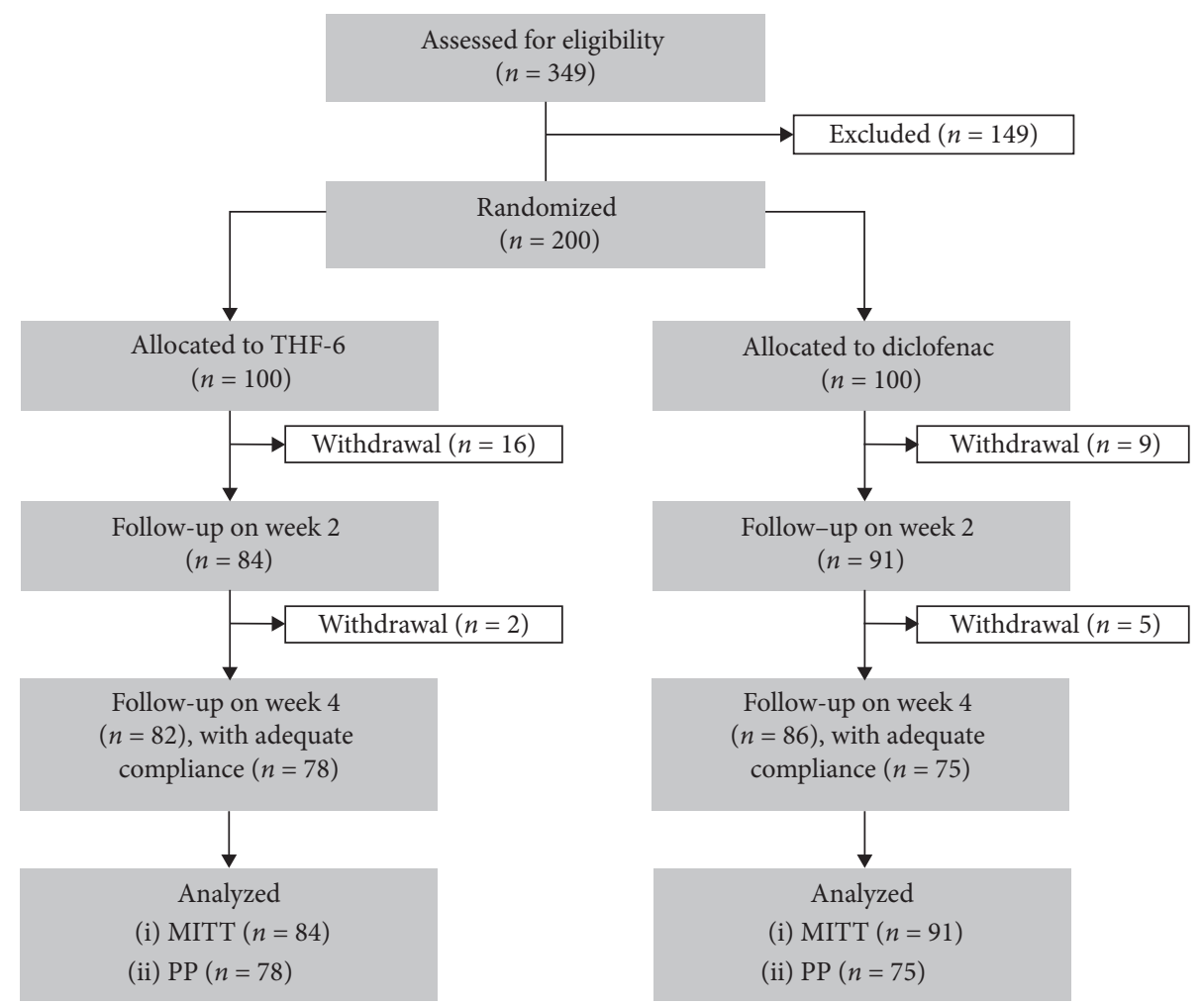

FIGURE 2: Flow diagram of the progress through all phases of this two-arm, randomized-controlled study (enrollment, intervention allocation, follow-up, and data analysis).

TABLe 1: Demographic and clinical characteristics of the enrolled patients.

\begin{tabular}{|c|c|c|}
\hline & THF-6 $(n=100)$ & Diclofenac $(n=100)$ \\
\hline Age (years) & $62.0 \pm 6.1$ & $60.3 \pm 6.1$ \\
\hline Female sex $(\%)$ & 87 & 84 \\
\hline BMI $\left(\mathrm{kg} / \mathrm{m}^{2}\right)$ & $26.0 \pm 4.7$ & $25.7 \pm 6.3^{\mathrm{a}}$ \\
\hline \multicolumn{3}{|l|}{ Location of osteoarthritis $(n)$} \\
\hline Right knee & 9 & 7 \\
\hline Left knee & 5 & 7 \\
\hline Both knees & 86 & 86 \\
\hline \multicolumn{3}{|l|}{ Kellgren-Lawrence grade $(n)$} \\
\hline Grade 2 & 62 & 68 \\
\hline Grade 3 & 68 & 73 \\
\hline Grade 4 & 56 & 45 \\
\hline Duration of osteoarthritis of the knee (years) & $4.9 \pm 4.7$ & $4.7 \pm 4.3$ \\
\hline \multicolumn{3}{|l|}{ Underlying disease $(n)$} \\
\hline Hypertension & 43 & 50 \\
\hline Dyslipidemia & 27 & 30 \\
\hline Diabetes mellitus & 13 & 12 \\
\hline Miscellaneous & 7 & 12 \\
\hline None & 49 & 37 \\
\hline \multicolumn{3}{|l|}{ Baseline measures } \\
\hline VAS pain $(\mathrm{mm})$ & $60.0 \pm 14.8$ & $61.9 \pm 15.7$ \\
\hline VAS stiffness $(\mathrm{mm})$ & $54.9 \pm 20.2$ & $56.9 \pm 22.3$ \\
\hline \multicolumn{3}{|l|}{ KOOS } \\
\hline Pain & $49.5 \pm 14.3$ & $51.4 \pm 17.1$ \\
\hline Other knee symptoms & $55.2 \pm 15.3$ & $53.7 \pm 18.3$ \\
\hline Activities of daily living & $51.3 \pm 16.7$ & $53.3 \pm 18.3$ \\
\hline Sport and recreation function & $24.4 \pm 19.4$ & $26.8 \pm 20.4$ \\
\hline Knee-related quality of life & $32.1 \pm 15.8$ & $32.0 \pm 17.6$ \\
\hline $\mathrm{SCT}(\mathrm{sec})$ & $12.1 \pm 5.9$ & $11.5 \pm 5.6$ \\
\hline
\end{tabular}

${ }^{a} n=99$. KOOS, Knee Injury and Osteoarthritis Outcome Score; SCT, 10-step stair climb test; VAS, visual analog scale. 
(87.5\%) were included in the MITT analysis and 153 (76.5\%) in the PP analysis (Figure 2).

With regard to the primary endpoint, the mean change in VAS pain was not statistically significantly different between the two groups, and THF-6 $(1,500 \mathrm{mg} /$ day $)$ was found to be noninferior to diclofenac $(75 \mathrm{mg} /$ day) in both the MITT and PP analyses. The upper limit of the two-sided 95\% CI for the comparison between the THF- 6 group and the diclofenac group was within the prespecified margin of $10 \mathrm{~mm}$ for noninferiority (Figure 3).

None of the mean changes in any of the efficacy outcome measures differed statistically significantly between the two groups (Table 2). Significant improvements in all efficacy parameters among patients receiving THF- 6 as well as those receiving oral diclofenac were observed by the end of Week 2 , and significant improvements continued to be seen at the end of Week 4 (Figures S1 and S2). There were 42 responders $(50 \%)$ at the end of the four-week treatment with THF-6 compared to $40(44 \%)$ with oral diclofenac (MITT analysis: $42 / 84$ vs. $40 / 91, p=0.423$; PP analysis: $39 / 78$ vs. $36 / 75$, $p=0.805)$. Overall improvement self-assessed by the patients was similar in both groups: no statistically significant difference between the two groups was observed at the end of the treatment phase (MITT analysis: $63.57 \pm 22.46$ vs. $64.20 \pm 22.92, \quad p=0.859$; PP analysis: $63.65 \pm 21.86$ vs. $63.00 \pm 23.71, p=0.859)$. Upon completion of this trial, the physician's assessment of overall improvement did not differ between the two groups (Figure S3).

Dyspepsia was the most common adverse event reported in both groups: 23 patients $(23 \%)$ in the THF- 6 group and $28(28 \%)$ in the diclofenac group (relative risk $=0.821,95 \% \mathrm{CI}=0.510$ to $1.323, p=0.417$ ). Overall, the proportion of patients reporting any adverse events in the safety population was fairly similar between the two groups (Table 3). All the adverse events reported were mild to moderate in intensity. Adverse events led to the premature discontinuation of 14 patients in the THF- 6 group and 12 patients in the diclofenac group $\left(\chi^{2}(1\right.$, $n=200)=0.177, p=0.674)$. Gastrointestinal intolerance was the major reason for discontinuation of therapy (9 in the THF- 6 group vs. 6 in the diclofenac group, $\chi^{2}(1$, $n=200)=0.649, p=0.421)$. During the trial, there was only one serious adverse event, hospitalization due to pesticide exposure, which was judged as definitely unrelated to the study drug.

\section{Discussion}

In this randomized-controlled trial, THF- 6 offered the potential to achieve analgesic efficacy with acceptable safety profiles in patients with moderate-to-severe osteoarthritis of the knee. In both the MITT and PP analyses, the upper limit of $95 \%$ CIs of MD in VAS pain was within the predefined noninferiority margin of $10 \mathrm{~mm}$. This allowed us to conclude that THF-6 was noninferior to oral diclofenac ( $75 \mathrm{mg} /$ day) in terms of knee pain relief. Oral administration of THF- 6 also resulted in significant improvement, comparable to oral diclofenac, across several outcome measures. As THF-6 and oral diclofenac displayed comparable efficacy and safety, it seems appropriate to consider THF- 6 an alternative for the treatment of moderate-to-severe osteoarthritis of the knee.

The present clinical trial was designed to evaluate the efficacy and safety of THF-6 versus oral diclofenac for the short-term treatment of symptomatic osteoarthritis of the knee. In clinical practice, pain relief medications, including oral NSAIDs, are typically prescribed on an as-needed basis for a short duration due to safety concerns [36]. Most randomized-controlled trials evaluating the effects of pharmacological interventions, especially oral NSAIDs, often last only a few weeks with only a small number of trials going beyond four weeks of treatment [26]. Therefore, the present trial design, with a treatment duration of four weeks, can be considered appropriate for determining the effects of THF-6 in symptomatic osteoarthritis of the knee.

With respect to osteoarthritic knee pain of moderate-tosevere intensity, this trial demonstrated that THF-6 was comparable to oral diclofenac in relieving pain symptoms. An average reduction of around $26 \mathrm{~mm}$ on a $100 \mathrm{~mm}$ VAS pain scale with THF-6 treatment can be considered a clinically important improvement [37]. A 50\% decrease in pain score, which represents a reasonable cut-off value for indicating a clinically meaningful pain reduction from the patient's perspective, was observed in approximately half of the patients in the THF-6 group [35]. These findings support the efficacy of THF- 6 in osteoarthritic knee pain relief.

Even though the precise mechanism of THF-6's action has not yet been elucidated, the herbal constituents in the formulation have been shown individually to possess several pharmacological activities, perceivably contributing to the beneficial effects of THF- 6 in the treatment of symptomatic osteoarthritis of the knee. Previous comprehensive literature reviews of $C$. rotundus have found that the plant extracts have a broad range of pharmacological activities, including anti-inflammatory, antinociceptive, antiarthritic, and antioxidant activities [38-40]. P. nigrum also exhibits various pharmacological activities, for example, anti-inflammatory, antinociceptive, antiarthritic, and antioxidant activities, in both in vitro and animal experiments [41-43]. S. asper contains a number of bioactive compounds which possess anti-inflammatory and antioxidant properties $[44,45]$. The crude extracts and isolated compounds of $T$. cordifolia have a wide range of pharmacological effects, including anti-inflammatory, antioxidant, antiarthritic, and analgesic properties [46, 47] as does $A$. procera [48-50]. As a result, it is reasonable to assume that the favorable outcomes following THF-6 administration in this trial might be attributable to the combined and possibly synergistic pharmacological activities of the several herbal ingredients in the formulation. Further research is warranted to ascertain the mechanisms of THF-6's action as well as understanding the molecular basis of its effects in symptomatic relief of osteoarthritic knee pain.

In this trial, the overall safety profiles of both pharmacological interventions were more or less the same. Around one-fourth of the patients in each group experienced gastrointestinal adverse events following drug administration. Dyspepsia was the most common adverse event in both groups and its frequency did not differ between 


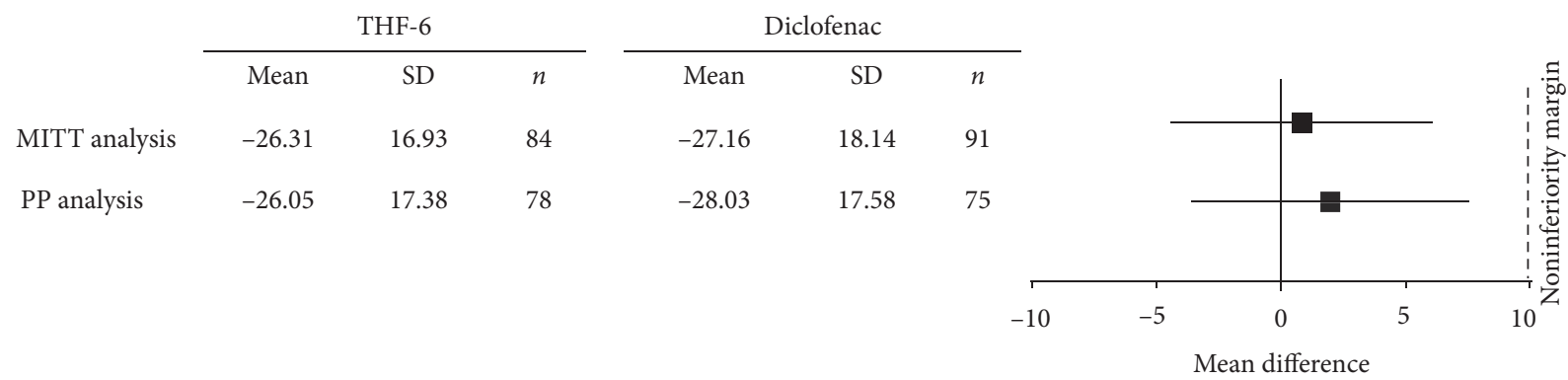

FIgURE 3: Noninferiority analysis of VAS pain.

TABle 2: Efficacy outcome assessments.

\begin{tabular}{|c|c|c|c|c|c|}
\hline & THF-6 & Diclofenac & Mean difference & $(95 \% \mathrm{CI})$ & $p$ value $^{\mathrm{a}}$ \\
\hline $\begin{array}{l}\text { Mean change of VAS pain }(\mathrm{mm}) \\
\text { MITT analysis } \\
\text { PP analysis }\end{array}$ & $\begin{array}{l}-26.31 \pm 16.93 \\
-26.05 \pm 17.38 \\
\end{array}$ & $\begin{array}{l}-27.16 \pm 18.14 \\
-28.03 \pm 17.58 \\
\end{array}$ & $\begin{array}{l}0.86 \\
1.98 \\
\end{array}$ & $\begin{array}{l}(-4.39 \text { to } 6.10) \\
(-3.61 \text { to } 7.56)\end{array}$ & $\begin{array}{l}0.748 \\
0.486 \\
\end{array}$ \\
\hline $\begin{array}{l}\text { Mean change of VAS stiffness }(\mathrm{mm}) \\
\text { MITT analysis } \\
\text { PP analysis }\end{array}$ & $\begin{array}{l}-22.52 \pm 17.70 \\
-22.53 \pm 17.82 \\
\end{array}$ & $\begin{array}{l}-23.26 \pm 19.12 \\
-23.28 \pm 18.10 \\
\end{array}$ & $\begin{array}{l}0.74 \\
0.75 \\
\end{array}$ & $\begin{array}{l}(-4.77 \text { to } 6.25) \\
(-4.98 \text { to } 6.49)\end{array}$ & $\begin{array}{l}0.791 \\
0.795\end{array}$ \\
\hline $\begin{array}{l}\text { Mean change of SCT }(\mathrm{sec}) \\
\text { MITT analysis } \\
\text { PP analysis }\end{array}$ & $\begin{array}{l}-3.35 \pm 3.55 \\
-3.44 \pm 3.61 \\
\end{array}$ & $\begin{array}{l}-3.42 \pm 4.33 \\
-3.27 \pm 4.09\end{array}$ & $\begin{array}{c}0.07 \\
-0.17 \\
\end{array}$ & $\begin{array}{l}(-1.12 \text { to } 1.26) \\
(-1.40 \text { to } 1.06) \\
\end{array}$ & $\begin{array}{l}0.148 \\
0.786 \\
\end{array}$ \\
\hline $\begin{array}{l}\text { Mean change of KOOS pain } \\
\text { MITT analysis } \\
\text { PP analysis }\end{array}$ & $\begin{array}{l}14.88 \pm 14.98 \\
15.24 \pm 15.29\end{array}$ & $\begin{array}{l}15.77 \pm 15.89 \\
15.77 \pm 14.54\end{array}$ & $\begin{array}{l}-0.89 \\
-0.53\end{array}$ & $\begin{array}{l}(-5.50 \text { to } 3.73) \\
(-5.30 \text { to } 4.24)\end{array}$ & $\begin{array}{l}0.705 \\
0.827\end{array}$ \\
\hline $\begin{array}{l}\text { Mean change of KOOS other symptoms } \\
\text { MITT analysis } \\
\text { PP analysis }\end{array}$ & $\begin{array}{l}12.29 \pm 14.92 \\
12.49 \pm 15.17 \\
\end{array}$ & $\begin{array}{l}15.29 \pm 16.90 \\
16.01 \pm 16.22 \\
\end{array}$ & $\begin{array}{l}-3.00 \\
-3.53 \\
\end{array}$ & $\begin{array}{l}(-7.77 \text { to } 1.77) \\
(-8.54 \text { to } 1.49)\end{array}$ & $\begin{array}{l}0.216 \\
0.167 \\
\end{array}$ \\
\hline $\begin{array}{l}\text { Mean change of KOOS activities of daily living } \\
\text { MITT analysis } \\
\text { PP analysis }\end{array}$ & $\begin{array}{l}13.25 \pm 15.22 \\
13.62 \pm 15.23\end{array}$ & $\begin{array}{l}14.46 \pm 17.78 \\
14.05 \pm 16.76 \\
\end{array}$ & $\begin{array}{l}-1.21 \\
-0.44 \\
\end{array}$ & $\begin{array}{l}(-6.17 \text { to } 3.75) \\
(-5.55 \text { to } 4.67)\end{array}$ & $\begin{array}{l}0.640 \\
0.866 \\
\end{array}$ \\
\hline $\begin{array}{l}\text { Mean change of KOOS sport and recreation function } \\
\text { MITT analysis } \\
\text { PP analysis }\end{array}$ & $\begin{array}{l}15.18 \pm 17.28 \\
15.64 \pm 17.48 \\
\end{array}$ & $\begin{array}{l}18.57 \pm 21.23 \\
19.27 \pm 21.79 \\
\end{array}$ & $\begin{array}{l}-3.39 \\
-3.63 \\
\end{array}$ & $\begin{array}{l}(-9.20 \text { to } 2.41) \\
(-9.91 \text { to } 2.66)\end{array}$ & $\begin{array}{l}0.250 \\
0.256 \\
\end{array}$ \\
\hline $\begin{array}{l}\text { Mean change of KOOS knee-related quality of life } \\
\text { MITT analysis } \\
\text { PP analysis }\end{array}$ & $\begin{array}{l}8.42 \pm 16.23 \\
9.38 \pm 16.30\end{array}$ & $\begin{array}{l}13.71 \pm 19.65 \\
13.95 \pm 18.01\end{array}$ & $\begin{array}{l}-5.30 \\
-4.56\end{array}$ & $\begin{array}{l}(-10.70 \text { to } 0.10) \\
(-10.05 \text { to } 0.92)\end{array}$ & $\begin{array}{l}0.054 \\
0.102\end{array}$ \\
\hline
\end{tabular}

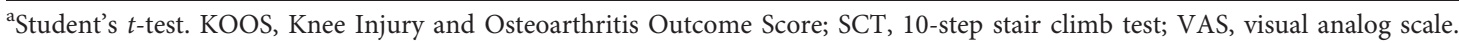

the groups. Nine patients in the THF-6 group discontinued treatment early due to gastrointestinal adverse consequences, as did six patients in the diclofenac group. These findings are not surprising given that similar gastrointestinal tolerability problems with oral diclofenac, particularly dyspepsia, have been regularly observed in other clinical trials assessing the safety and tolerability of oral NSAIDs $[51,52]$. Based on its safety profile as described above, THF-6 should be used with caution, especially in patients at high risk of upper gastrointestinal complications.

There were limitations to this trial. First and foremost, there was no placebo comparison in the trial where a subjective outcome measure was used as a primary endpoint. In the context where standard therapy is widely used to treat a condition, a placebo comparison might not be possible due primarily to ethical reasons [53]. In such a context, noninferiority design could be applied to compare the intervention under investigation with another active treatment provided that the reference treatment's efficacy is well established [54], as is the case for oral diclofenac [9]. This is of particular relevance in the present trial where the target groups had baseline knee pain of moderate-to-severe intensity. To minimize the risk of bias in the assessment of subjective outcomes, this trial assessed knee symptoms using several different measures to help ensure a comprehensive evaluation and also included a 10-step SCT measure which is less subject to contextual effects [55]. It should also be acknowledged that the MITT approach may be prone to attrition bias associated with the exclusion of some patients from the analysis [56, 57]. Notwithstanding, it does not necessarily bias trial results [58]. In noninferiority trials, a PP analysis typically yields a more conservative estimate of treatment effects; therefore, the MITT approach is less likely to have a significant impact on estimated treatment benefits especially when both PP and MITT analyses consistently support noninferiority, as is the case of the present study 
TABLE 3: Safety outcome assessments.

\begin{tabular}{|c|c|c|c|}
\hline Adverse events & THF-6 $(n=100)$ & Diclofenac $(n=100)$ & $p$ value $^{\mathrm{a}}$ \\
\hline \multicolumn{4}{|l|}{ Gastrointestinal system } \\
\hline Dyspepsia & 23 & 28 & 0.417 \\
\hline Nausea and vomiting & 3 & 4 & 1.000 \\
\hline Gastroesophageal reflux disease & 2 & 2 & 1.000 \\
\hline Loss of appetite & 4 & 1 & 0.369 \\
\hline Diarrhea & 3 & 1 & 0.621 \\
\hline Constipation & 5 & 5 & 1.000 \\
\hline Hematochezia & 1 & 0 & 1.000 \\
\hline \multicolumn{4}{|l|}{ Other systems } \\
\hline Peripheral edema & 4 & 5 & 1.000 \\
\hline Peripheral neuropathy & 2 & 1 & 1.000 \\
\hline Dizziness & 1 & 2 & 1.000 \\
\hline Headache & 1 & 0 & 1.000 \\
\hline Drowsiness & 1 & 0 & 1.000 \\
\hline Insomnia & 0 & 1 & 1.000 \\
\hline Dry mouth & 0 & 1 & 1.000 \\
\hline Blurred vision & 1 & 0 & 1.000 \\
\hline Low back pain & 2 & 2 & 1.000 \\
\hline Myalgia & 2 & 0 & 0.497 \\
\hline Cramp & 1 & 1 & 1.000 \\
\hline Rashes & 2 & 0 & 0.497 \\
\hline Itching & 2 & 0 & 0.497 \\
\hline AST elevation ( $>2.5-3.0$ times from baseline) & 2 & 1 & 1.000 \\
\hline Creatinine rising ( $>1.5-2.0$ times from baseline) & 0 & 3 & 0.246 \\
\hline
\end{tabular}

${ }^{a}$ Chi-square test or Fisher's exact test. AST: aspartate aminotransferase.

[59]. Lastly, it is notable that the trial length of four weeks may not be long enough to determine long-term adverse outcomes of THF-6, including cardiovascular complications [60]. On the basis of the evidence currently available, this trial does not guarantee the safety of long-term use of THF6.

\section{Conclusions}

THF-6 offers an alternative to oral diclofenac for the shortterm treatment of moderate-to-severe osteoarthritis of the knee. Administration of THF- 6 was shown to be noninferior to oral diclofenac in relieving knee pain. None of the outcome measures assessed in this trial favored either THF-6 or oral diclofenac with respect to symptomatic osteoarthritis of the knee.

\section{Data Availability}

All data used to support the findings of this study are available from the corresponding author upon reasonable request.

\section{Conflicts of Interest}

The authors declare no conflicts of interest regarding the publication of this article.

\section{Authors' Contributions}

NK, SN, ST, NC, CS, SC, and NH contributed to study conception and design. SN and $\mathrm{NH}$ developed the study protocol and related documents. SC supervised a manufacturing process of herbal preparations and placebo. PT contributed to the HPLC method development. SN and $\mathrm{NH}$ conducted the study and collected data. NK analyzed the data. NK and NH interpreted the results. NK drafted and finalized the manuscript with support and comments from ST, SN, NC, CS, SC, and NH. All authors read and approved the final manuscript.

\section{Acknowledgments}

The authors are grateful for the editorial assistance of Dr. G. Lamar Robert, English language consultant to the Department of Pharmacology, Faculty of Medicine, Chiang Mai University. The authors would also like to extend their thanks to Ms. Sujitra Techatoei for her secretarial assistance. This work was supported by the Department of Thai Traditional and Alternative Medicine, Ministry of Public Health.

\section{Supplementary Materials}

Table S1: components of Thai herbal recipe-6 (THF-6). Figure S1: VAS pain, VAS stiffness, and SCT at baseline, Week 2, and Week 4. Figure S2: KOOS at baseline, Week 2, and Week 4. Figure S3: Patient's and physician's opinion of overall improvement. (Supplementary Materials)

\section{References}

[1] D. J. Hunter and S. Bierma-Zeinstra, "Osteoarthritis," The Lancet, vol. 393, no. 10182, pp. 1745-1759, 2019. 
[2] M. Kloppenburg and F. Berenbaum, "Osteoarthritis year in review 2019: epidemiology and therapy," Osteoarthritis and Cartilage, vol. 28, no. 3, pp. 242-248, 2020.

[3] M. Cross, E. Smith, D. Hoy et al., "The global burden of hip and knee osteoarthritis: estimates from the Global Burden of Disease 2010 study," Annals of the Rheumatic Diseases, vol. 73, no. 7, pp. 1323-1330, 2014.

[4] GBD 2015 DALYs and HALE Collaborators, "Global, regional, and national disability-adjusted life-years (DALYs) for 315 diseases and injuries and healthy life expectancy (HALE), 1990-2015: a systematic analysis for the Global burden of disease study 2015," Lancet, vol. 388, no. 10053, pp. 16031658, 2016.

[5] GBD 2017 DALYs and HALE Collaborators, "Global, regional, and national disability-adjusted life-years (DALYs) for 359 diseases and injuries and healthy life expectancy (HALE) for 195 countries and territories, 1990-2017: a systematic analysis for the Global burden of disease study 2017," Lancet, vol. 392, no. 10159, pp. 1859-1922, 2018.

[6] L. Murphy, T. A. Schwartz, C. G. Helmick et al., "Lifetime risk of symptomatic knee osteoarthritis," Arthritis \& Rheumatism, vol. 59, no. 9, pp. 1207-1213, 2008.

[7] D. J. Hunter, D. Schofield, and E. Callander, "The individual and socioeconomic impact of osteoarthritis," Nature Reviews Rheumatology, vol. 10, no. 7, pp. 437-441, 2014.

[8] S. R. Kingsbury, E. M. Hensor, C. A. Walsh, M. C. Hochberg, and P. G. Conaghan, "How do people with knee osteoarthritis use osteoarthritis pain medications and does this change over time? data from the osteoarthritis Initiative," Arthritis Research \& Therapy, vol. 15, no. 5, p. R106, 2013.

[9] B. R. da Costa, S. Reichenbach, N. Keller et al., "Effectiveness of non-steroidal anti-inflammatory drugs for the treatment of pain in knee and hip osteoarthritis: a network meta-analysis," The Lancet, vol. 390, no. 10090, pp. e21-e33, 2017.

[10] R. R. Bannuru, M. C. Osani, E. E. Vaysbrot et al., "OARSI guidelines for the non-surgical management of knee, hip, and polyarticular osteoarthritis," Osteoarthritis and Cartilage, vol. 27, no. 11, pp. 1578-1589, 2019.

[11] S. L. Kolasinski, T. Neogi, M. C. Hochberg et al., “2019 American college of rheumatology/arthritis foundation guideline for the management of osteoarthritis of the hand, hip, and knee," Arthritis Care \& Research, vol. 72, no. 2, pp. 149-162, 2020.

[12] J. Hasford, N. Moore, and K. Hoye, "Safety and usage pattern of low-dose diclofenac when used as an over-the-counter medication: results of an observational cohort study in a community-based pharmacy setting," International Journal of Clinical Pharmacology and Therapeutics, vol. 42, no. 08, pp. 415-422, 2004.

[13] N. Moore and J. M. Scheiman, "Gastrointestinal safety and tolerability of oral non-aspirin over-the-counter analgesics," Postgraduate Medicine, vol. 130, no. 2, pp. 188-199, 2018.

[14] K. J. Foreman, N. Marquez, A. Dolgert et al., "Forecasting life expectancy, years of life lost, and all-cause and cause-specific mortality for 250 causes of death: reference and alternative scenarios for 2016-40 for 195 countries and territories," The Lancet, vol. 392, no. 10159, pp. 2052-2090, 2018.

[15] N. Koonrungsesomboon, S. Teekachunhatean, S. Chansakaow, and N. Hanprasertpong, "Clinical efficacy and safety of yellow oil formulations 3 and 4 versus indomethacin solution in patients with symptomatic osteoarthritis of the knee: a randomized controlled trial," Evidence-Based Complementary and Alternative Medicine, vol. 2020, Article ID 5782178, , 2020.
[16] D. Dragos, M. Gilca, L. Gaman et al., "Phytomedicine in joint disorders," Nutrients, vol. 9, no. 1, p. 70, 2017.

[17] M. Cameron and S. Chrubasik, "Oral herbal therapies for treating osteoarthritis," Cochrane Database Syst Rev, vol. 5, no. 5, Article ID CD002947, 2014.

[18] B. Chen, H. Zhan, J. Marszalek et al., "Traditional Chinese medications for knee osteoarthritis pain: a meta-analysis of randomized controlled trials," The American Journal of Chinese Medicine, vol. 44, no. 04, pp. 677-703, 2016.

[19] F. Wang, L. Shi, Y. Zhang et al., "A traditional herbal formula Xianlinggubao for pain control and function improvement in patients with knee and hand osteoarthritis: a multicenter, randomized, open-label, controlled trial," Evidence-Based Complementary and Alternative Medicine, vol. 2018, Article ID 1827528, 2018.

[20] C. Chailom, Pramuan Tamrap Ya Thai, Sripanya, Nonthaburi, Thailand, in Thai, 2011.

[21] P. W. Rattanarangsri, Petnamnueng Khong Borannachan, Odeon Store, Bangkok, Thailand, in Thai, 1991.

[22] S. Pitiphon, "Samunphrai Abhaibhubejhr Suebsab Phum Phanya Thaid," Pauramutha Karnpim Co., Ltd., Bangkok, Thailand, in Thai, 2004.

[23] T. E. McAlindon, J. B. Driban, Y. Henrotin et al., "OARSI clinical trials recommendations: design, conduct, and reporting of clinical trials for knee osteoarthritis," Osteoarthritis and Cartilage, vol. 23, no. 5, pp. 747-760, 2015.

[24] R. Altman, E. Asch, D. Bloch et al., "Development of criteria for the classification and reporting of osteoarthritis: classification of osteoarthritis of the knee," Arthritis \& Rheumatism, vol. 29, no. 8, pp. 1039-1049, 1986.

[25] A. M. Boonstra, H. R. Schiphorst Preuper, G. A. Balk, and R. E. Stewart, "Cut-off points for mild, moderate, and severe pain on the visual analogue scale for pain in patients with chronic musculoskeletal pain," Pain, vol. 155, no. 12, pp. 2545-2550, 2014.

[26] J. M. Bjordal, A. Klovning, A. E. Ljunggren, and L. Slordal, "Short-term efficacy of pharmacotherapeutic interventions in osteoarthritic knee pain: a meta-analysis of randomised placebo-controlled trials," European Journal of Pain, vol. 11, no. 12, pp. 125-138, 2007.

[27] A. van Walsem, S. Pandhi, R. M. Nixon et al., "Relative benefit-risk comparing diclofenac to other traditional nonsteroidal anti-inflammatory drugs and cyclooxygenase- 2 inhibitors in patients with osteoarthritis or rheumatoid arthritis: a network meta-analysis," Arthritis Research \& Therapy, vol. 17, no. 1, p. 66, 2015.

[28] S. A. Julious, "Sample sizes for clinical trials with normal data," Statistics in Medicine, vol. 23, no. 12, pp. 1921-1986, 2004.

[29] S. R. Kingsbury, N. Corp, F. E. Watt et al., "Harmonising data collection from osteoarthritis studies to enable stratification: recommendations on core data collection from an arthritis research UK clinical studies group," Rheumatology, vol. 55, no. 8, pp. 1394-1402, 2016.

[30] T. O. Smith, G. A. Hawker, D. J. Hunter et al., "The OMERACT-OARSI core domain set for measurement in clinical trials of hip and/or knee osteoarthritis," The Journal of Rheumatology, vol. 46, no. 8, pp. 981-989, 2019.

[31] G. A. Hawker, S. Mian, T. Kendzerska, and M. French, "Measures of adult pain: visual analog scale for pain (VAS pain), numeric rating scale for pain (NRS pain), McGill pain questionnaire (MPQ), short-form McGill pain questionnaire (SF-MPQ), chronic pain grade scale (CPGS), short form-36 
bodily pain scale (SF)," Arthritis Care \& Research, vol. 63, no. S11, pp. S240-S252, 2011.

[32] K. Bennell, F. Dobson, and R. Hinman, "Measures of physical performance assessments: self-paced walk test (SPWT), stair climb test (SCT), six-minute walk test (6MWT), chair stand test (CST), timed up \& go (TUG), sock test, lift and carry test (LCT), and car task," Arthritis Care \& Research, vol. 63, no. S11, pp. S350-S370, 2011.

[33] N. J. Collins, D. Misra, D. T. Felson, K. M. Crossley, and E. M. Roos, "Measures of knee function: international knee documentation committee (IKDC) subjective knee evaluation form, knee injury and osteoarthritis outcome score (KOOS), knee injury and osteoarthritis outcome score physical function short form (KOOS-PS), knee ou," Arthritis Care \& Research, vol. 63, no. S11, pp. S208-S228, 2011.

[34] K. Chaipinyo, "Test-retest reliability and construct validity of Thai version of knee osteoarthritis outcome score (KOOS)," Thai Journal of Physical Therapy Science, vol. 31, pp. 67-76, 2009.

[35] T. Pham, D. van der Heijde, R. D. Altman et al., "OMERACTOARSI Initiative: osteoarthritis Research Society International set of responder criteria for osteoarthritis clinical trials revisited," Osteoarthritis and Cartilage, vol. 12, no. 5, pp. 389-399, 2004.

[36] C. Cooper, R. Chapurlat, N. Al-Daghri et al., "Safety of oral non-selective non-steroidal anti-inflammatory drugs in osteoarthritis: what does the literature say?" Drugs Aging, vol. 36, no. 1, pp. 15-24, 2019.

[37] F. Tubach, P. Ravaud, G. Baron et al., "Evaluation of clinically relevant changes in patient reported outcomes in knee and hip osteoarthritis: the minimal clinically important improvement," Annals of the Rheumatic Diseases, vol. 64, no. 1, pp. 29-33, 2005.

[38] A. E. Al-Snafi, "A review on Cyperus rotundus A potential medicinal plant," IOSR Journal of Pharmacy (IOSRPHR), vol. 6, no. 7, pp. 32-48, 2016.

[39] N. Singh, B. R. Pandey, P. Verma et al., "Phyto-pharmacotherapeutics of Cyperus rotundus Linn. (Motha): an overview," Indian Journal of Natural Products and Resources, vol. 3, no. 4, pp. 467-476, 2012.

[40] S. R. Sivapalan, "Medicinal uses and pharmacological activities of Cyperus rotundus Linn-a review," International Journal of Science and Research, vol. 3, no. 5, pp. 1-8, 2013.

[41] J. S. Bang, D. H. Oh, H. M. Choi et al., "Anti-inflammatory and antiarthritic effects of piperine in human interleukin $1 \beta$ stimulated fibroblast-like synoviocytes and in rat arthritis models," Arthritis Research \& Therapy, vol. 11, no. 2, p. R49, 2009.

[42] M. Meghwal and T. K. Goswami, "Piper nigrum and piperine: an update," Phytotherapy Research, vol. 27, no. 8, pp. 1121-1130, 2013.

[43] F. Tasleem, I. Azhar, S. N. Ali et al., "Analgesic and antiinflammatory activities of Piper nigrum L," Asian Pacific Journal of Tropical Medicine, vol. 7, no. Suppl 1, pp. S461S468, 2014.

[44] N. Ibrahim, I. Mat, V. Lim, and R. Ahmad, “Antioxidant activity and phenolic content of Streblus asper leaves from various drying methods," Antioxidants, vol. 2, no. 3, pp. 156-166, 2013.

[45] B. Sripanidkulchai, J. Junlatat, N. Wara-aswapati, and D. Hormdee, "Anti-inflammatory effect of Streblus asper leaf extract in rats and its modulation on inflammation-associated genes expression in RAW 264.7 macrophage cells," Journal of Ethnopharmacology, vol. 124, no. 3, pp. 566-570, 2009.
[46] S. Saha and S. Ghosh, "Tinospora cordifolia: one plant, many roles," Ancient Science of Life, vol. 31, no. 4, pp. 151-159, 2012.

[47] S. Upadhayay, M. Bora, L. Kawlni et al., "Comprehensive pharmacology review of guduchi [Tinospora cordifolia (wild.) Miers]," Journal of Drug Research in Ayurvedic Sciences, vol. 3, no. 1, pp. 48-52, 2018.

[48] M. M. Khatoon, M. H. Khatun, M. E. Islam, and M. S. Parvin, "Analgesic, antibacterial and central nervous system depressant activities of Albizia procera leaves," Asian Pacific Journal of Tropical Biomedicine, vol. 4, no. 4, pp. 279-284, 2014.

[49] K. Kokila, S. D. Priyadharshini, and V. Sujatha, "Phytopharmacological properties of Albizia species: a review," International Journal of Pharmacy and Pharmaceutical Sciences, vol. 5, no. 3, pp. 70-73, 2013.

[50] S. Sivakrishnan and A. Kottai Muthu, "In vivo antioxidant activity of ethanolic extract of aerial parts of Albizia procera roxb (benth.) against paracetamol induced liver toxicity on Wistar rats," Journal of Pharmaceutical Sciences and Research, vol. 5, no. 9, pp. 174-177, 2013.

[51] Coxib and traditional NSAID Trialists' (CNT) Collaboration, N. Bhala, J. Emberson et al., "Vascular and upper gastrointestinal effects of non-steroidal anti-inflammatory drugs: meta-analyses of individual participant data from randomised trials," Lancet, vol. 382, no. 9894, pp. 769-779, 2013.

[52] S. R. Mallen, M. N. Essex, and R. Zhang, "Gastrointestinal tolerability of NSAIDs in elderly patients: a pooled analysis of 21 randomized clinical trials with celecoxib and nonselective NSAIDs," Current Medical Research and Opinion, vol. 27, no. 7, pp. 1359-1366, 2011.

[53] A. S. Skierka and K. B. Michels, "Ethical principles and placebo-controlled trials-interpretation and implementation of the declaration of Helsinki's placebo paragraph in medical research," BMC Med Ethics, vol. 19, no. 1, p. 24, 2018.

[54] L. Mauri and R. B. D'Agostino, "Challenges in the design and interpretation of noninferiority trials," New England Journal of Medicine, vol. 377, no. 14, pp. 1357-1367, 2017.

[55] F. Dobson, R. S. Hinman, E. M. Roos et al., "OARSI recommended performance-based tests to assess physical function in people diagnosed with hip or knee osteoarthritis," Osteoarthritis and Cartilage, vol. 21, no. 8, pp. 1042-1052, 2013.

[56] I. Abraha and A. Montedori, "Modified intention to treat reporting in randomised controlled trials: systematic review," BMJ, vol. 340, p. c2697, 2010.

[57] E. Nuesch, S. Trelle, S. Reichenbach et al., "The effects of excluding patients from the analysis in randomised controlled trials: meta-epidemiological study," BMJ, vol. 339, p. b3244, 2009.

[58] A. Dossing, S. Tarp, D. E. Furst et al., "Modified intention-totreat analysis did not bias trial results," Journal of Clinical Epidemiology, vol. 72, pp. 66-74, 2016.

[59] S. J. Oczkowski, "A clinician's guide to the assessment and interpretation of noninferiority trials for novel therapies," Open Medicine: A Peer-Reviewed, Independent, Open-Access Journal, vol. 8, no. 2, pp. e67-72, 2014.

[60] C. A. Barcella, M. Lamberts, P. McGettigan et al., "Differences in cardiovascular safety with non-steroidal anti-inflammatory drug therapy-a nationwide study in patients with osteoarthritis," Basic \& Clinical Pharmacology \& Toxicology, vol. 124, no. 5, pp. 629-641, 2019. 